\title{
Higher log position is not associated with better physical fitness in professional soccer teams in South Africa
}

\author{
J R Clark (BSc Hons), BA (Hons), CSCS \\ Institute for Sport Research, Department of Biokinetics, Sport and Leisure Sciences, University of Pretoria
}

\begin{abstract}
Objective. To assess the difference in physical fitness of players in successful versus less-successful professional soccer teams in South Africa.

Design. Professional soccer players $(N=140)$ underwent a battery of tests assessing important physiological components during the early part of their competitive season. Players were then separated into two groups on the basis of their teams' final log position in the Premier Soccer League (PSL) in South Africa. Players in successful ( $N=$ $70)$ and less-successful $(N=70)$ teams were in the top four or bottom six positions on the log respectively. Descriptive statistics (mean \pm standard deviation (SD)) were calculated for each group, and independent $t$-tests were used to compare the means of the groups for each of the physical tests.
\end{abstract}

Main outcome measures. Body composition, flexibility, muscle strength-endurance, power, speed, agility, aerobic endurance, and repeat sprint distance.

Results. There were no significant differences between groups for all measures of body composition, flexibility, repeat sprint distance, and agility. Significant differences $(p<0.05)$ were found for sit-ups, aerobic endurance, and speed, but these were generally small, not meaningful differences in performance. Players in successful squads were significantly $(p<0.01)$ older than those in less-successful teams.

Conclusions. The results demonstrate that in South Africa level of physical fitness is not higher in more-successful compared with less-successful teams in the PSL. Factors other than physical fitness may be more important in determining successful league performance and discrimi-

\section{CORRESPONDENCE:}

\section{J R Clark}

Institute for Sport Research

LC de Villiers Sport Centre

University of Pretoria

0002

Tel: 012-420 6033

Fax: 012-420 6099

E-mail: jimmy.clark@up.ac.za nate better between players in teams with different levels of success. Improving professional soccer performance may require coaches and trainers to focus more attention on technical and tactical skill development in sport-specific training once an acceptable standard of fitness has been attained.

\section{Introduction}

In 2010 South Africa will host the soccer world cup for the first time on the African continent. In 1996 the South African national team was ranked 16 th in the world. Ten years later the South African team was ranked 69th. This gradual slide in ranking has led to apprehension over South Africa's performance in the next world cup finals. ${ }^{1}$ Part of the perennial public criticism of many South African national team performances concerns the physical fitness of players. For sport scientists to make meaningful contributions to team performance, objective data are needed related to these phenomena.

Soccer is the most widely played and watched sport in the world. ${ }^{12,27}$ Mirroring its global popularity is a vast amount of scientific enquiry devoted to soccer. This has included studies on player motion analyses, metabolic profiling of match requirements, anthropometrical and fitness testing and technical skill assessments, ${ }^{19}$ involving both elite and non-elite, ${ }^{22}$ youth and senior, ${ }^{5}$ and male and female players, ${ }^{6}$ and even soccer referees. ${ }^{23}$

As with most sports, a myriad physical, psychological and technical factors contribute to performance. ${ }^{2,3,14,23}$ It is generally accepted that a wide range of physical fitness attributes are vital in soccer, particularly in the modern game. $^{22,24}$ Players' physical capacities are even said to contribute to the technical and tactical skills in soccer. ${ }^{5,23}$ Improved physical capacity had a positive effect on distance covered, number of sprints, and involvement in decisive plays during a soccer match. ${ }^{14}$ Some researchers report that performance in fitness testing is related to the level of playing ability. ${ }^{14,20,23,26}$ Furthermore, Svensson and Drust ${ }^{24}$ report that physical performance is an important consideration in player selection.

Both laboratory and field tests have been used extensively to assess soccer players' physical performance at both amateur and elite levels. ${ }^{24}$ Physiological testing may be useful in determining individual strengths and weakness, monitoring changes in training status, and guiding further exercise prescription. ${ }^{17}$ Tests are frequently performed at the 
start and end of the pre-season to evaluate the effectiveness of training preparation. ${ }^{24}$ Although field tests may provide less direct and accurate measurements than laboratory tests, they have greater specificity. ${ }^{17}$

Sports performance is frequently regarded as a function of genetic endowment, training and health status, and athlete skill, in various combinations. ${ }^{17}$ Sport scientists and trainers are often tasked with maximising physical performance with the aim of improving competition success. Indeed, some authors have recommended that, at least in European leagues, more focus be directed towards the effective training of players' physical abilities. ${ }^{23}$ The extent to which technical/ tactical versus physical fitness interventions are required remains a difficult question to answer in practice. Are similar recommendations justified in a South African context? Just how fit a team needs to be to achieve success is important in deciding on coaching and training schedules, training session focus, and player selection. More specifically, the question of whether physical fitness of players is a contributing factor to the difference between successful and less-successful teams in South Africa should be addressed.

Fitness test performance may vary depending on the individual player's profile, their position of play, and the team's style of play. ${ }^{19,23,24}$ It has been reported that the work rates of elite players are higher than those of non-elite players, and that this is achieved by both higher aerobic and anaerobic rates of metabolism. ${ }^{23}$ Yet most researchers agree that individual test results cannot be used to conclusively predict performance in match-play due to the complex requirements for soccer success. ${ }^{24}$ It is less clear whether team success is associated with the overall physical fitness of the team. Stølen et al. $^{23}$ reported that the lowest ranked national teams had lower maximal oxygen consumption $\left(\mathrm{VO}_{2 \max }\right)$ values than the best national teams. Hoff ${ }^{14}$ also reported that a relationship exists between average $\mathrm{VO}_{2 \max }$ and team performance in European squads.

The purpose of this study was therefore to assess whether there were significant differences in physical fitness between professional soccer players in South African teams placed high and low on the league log. Results would be useful for coaching, technical and conditioning staff in directing the allocation of training time. This is particularly important in an era in which competition schedules are increasingly demanding, and available preparation time needs to be used most effectively.

\section{Methods}

\section{Subjects}

One-hundred-and-forty players from professional teams in the Premier Soccer League (PSL) in South Africa were recruited for the study. The physical testing formed part of the ongoing physical assessment programmes for the teams. Only players declared medically fit to participate in all the testing by the team doctors were included. All players were briefed on the purpose, benefits and risks involved in the testing, and all completed written informed consent forms.
Subjects were assigned to one of two groups based on their teams' final league position at the end of the season in which they were tested. Teams were classified as successful or unsuccessful by finishing in the top four or bottom six positions of the PSL log, respectively.

\section{Procedures}

Data collection took place at the Institute for Sport Research, University of Pretoria, during the months of October and November in 2005 and 2006. This falls in the first half of the domestic competitive soccer season, after all teams had been through pre-season training in June and July, and had started the new season in August. This period of the season was marked by 4 - 7 training sessions per week, of which 3 - 5 involved focused physical conditioning of 45 - 90 minutes per session.

All testing occurred in a team format, with group instruction and explanation before testing, and strong verbal motivation of players during the tests. Testing sessions were completed in a single day on each occasion, with teams being tested on different days. Testing was always conducted between $08 \mathrm{~h} 00$ and $12 \mathrm{~h} 00$, in the same test order, and with the same rest periods between consecutive tests. Anthropometrical, flexibility, and muscle strength-endurance assessments were conducted indoors in a temperature-controlled $\left(\sim 21^{\circ} \mathrm{C}\right)$ setting. All other tests were conducted outdoors, with maximum ambient temperatures during testing ranging from $24^{\circ} \mathrm{C}$ to $27^{\circ} \mathrm{C}$. Subjects were instructed to arrive for the testing well rested, well hydrated and fed, to avoid caffeinecontaining foods on the day of testing, and to avoid physical exercise on the day prior to the testing. Subjects had access to water through the duration of the testing.

A standardised general warm-up was administered to the team prior to the outdoor testing. This lasted 15 minutes and consisted of easy running, stretching, dynamic drills, and harder but submaximal acceleration sprints. More specific warm-up and familiarisation drills took place immediately before each of the subsequent tests.

\section{Anthropometry and body composition}

The anthropometrical measuring procedures described by Norton et al. ${ }^{18}$ were used. Body mass was measured using a Tanita BF-350 electronic scale (Tanita Corporation, Tokyo, Japan) and players' stature was measured using a Seca 214 stadiometer (Seca Corporation, Hanover, USA). Skinfold thickness (triceps, subscapular, biceps, supra-iliac, calf, thigh, and abdominal) was measured using a Harpenden skinfold caliper (Baty International, British Indicators, West Sussex, England). These were summed to obtain the sum of seven skinfolds. ${ }^{18}$ Percentage body fat was estimated from these measures by predicting body density from the equation by Durnin and Womersley ${ }^{7}$ and then estimating per cent body fat based on the Siri formula as described by Lohman. ${ }^{16}$

\section{Flexibility}

The modified sit-and-reach test ${ }^{13}$ was used to assess hip and trunk flexion ${ }^{9}$ using a sit-and-reach box. Players were in- 
structed to stretch their hamstrings and low back prior to the test, after which test procedures were followed as described by Hoeger. ${ }^{13}$ The total displacement of the fingertips between reach and stretch distance was recorded to the nearest 0.5 $\mathrm{cm}$, and the best of three trials was accepted as the final score.

\section{Strength-endurance}

Strength-endurance measures included overhand pull-ups, bent-knee sit-ups, and push-ups. Maximum pull-ups were assessed with players maintaining a pronated (overhand) grip on a fixed overhead, wall-mounted pull-up bar. Hands were placed $5 \mathrm{~cm}$ wider than shoulder width. Pulling the body up from a hanging, straight-arm position to end with the chin above the bar was considered a legitimate pull-up. Players performed the maximum number of repetitions possible without touching the wall or floor.

Sit-ups were performed with knees bent at $90^{\circ}$ and feet secured to the floor. With arms crossed on the chest and hands holding the shoulders, players curled up from a supine position until the elbows touched the knees, and descended until the scapula touched the floor. Players performed the maximum number of sit-ups in 2 minutes.

The maximum number of push-ups that players could perform in 1 minute was measured. Players assumed a prone position with thumbs shoulder-width apart. A legitimate push-up involved pressing the body upward until the elbows were extended with simultaneous ascent of the hips and shoulders. Descent required lowering the body with the arms until the elbows were bent to $90^{\circ}$ while only the hands and the toes touched the floor.

\section{Power}

Jumping ability incorporating explosive knee and hip extension was assessed through the vertical jump test. ${ }^{9}$ After the generalised warm-up described above, players were instructed to perform light body-weight squats and submaximal jumps in preparation for the maximal-effort jumps, before instruction on test procedure. A Vertec device (Sports Imports, Columbus, USA) was securely set on a hard level surface. Players stood side-on to the device with their right shoulder in line with the vanes. The test was conducted using the procedures described by Ellis et al., ${ }^{9}$ and the greatest distance between reach and jump height was recorded to the nearest $1 \mathrm{~cm}$ after three trials. Players rested between efforts. The athletes' power output was estimated using the Lewis formula using body mass and vertical jump distance ${ }^{10}$ and then divided by body mass to obtain relative power output in $\mathrm{W} / \mathrm{kg}$.

\section{Speed}

A maximum-effort sprint running test was used to assess players' speed and acceleration from a stationary position. Subjects sprinted on a level, even surface, in a straight line on a natural grass soccer pitch with the subjects wearing full soccer kit. Players were briefed on the start procedure and allowed 2 - 3 submaximal 'acceleration sprints' along the test distance. The Swift Speedlight Timing System (Alstonville, Australia) was used in conjunction with the protocol described by Ellis et al. ${ }^{9}$ Timing gates were placed at chest height at $0 \mathrm{~m}, 10 \mathrm{~m}$, and $40 \mathrm{~m}$ intervals along a straight line. Players started $30 \mathrm{~cm}$ behind the $0 \mathrm{~m}$ mark from a standing start when they were ready, initiating the timer on crossing the $0 \mathrm{~m}$ mark, thus eliminating reaction time. The fastest of two trials was recorded to the nearest $0.01 \mathrm{~s}$ for $10 \mathrm{~m}$ and 40 $\mathrm{m}$. Players rested for around 5 minutes between attempts.

\section{Agility}

The Illinois agility test, adapted and modified by Roozen ${ }^{21}$ and Tossavainen ${ }^{25}$ from Getchell ${ }^{11}$ was used as a measure of agility. The test requires maximal effort acceleration, deceleration and direction change while sprinting between a grid of cones. A level, even surface on a natural grass soccer pitch was used for the test, with the subjects wearing full soccer kit. Subjects performed a fast but submaximal run in the required pattern before the first test effort. The Swift Speedlight Timing System (Alstonville, Australia) was used to measure test time with a timing gate set up at the finish line. Players started in the prone position behind the start line. Timing was initiated by an audio signal whereupon players got up and sprinted through the predetermined grid of cones. ${ }^{25}$ The fastest time of two attempts was recorded. Players had about 5 minutes of rest between attempts.

\section{Aerobic endurance}

The progressive maximal $20 \mathrm{~m}$ Multi-stage Shuttle run Test (MST) was used to assess aerobic power. ${ }^{15}$ This test has been widely used to test English football players ${ }^{24}$ and is recommended as a test of aerobic endurance by Stølen et al. ${ }^{23}$ The test was conducted on a non-slip hard court surface. ${ }^{15}$ Players were instructed to pace their runs along the $20 \mathrm{~m}$ shuttle distance according to the audio signal from the recorded compact disc. Failure to maintain the required pace for two consecutive shuttles constituted a criterion for a verbal warning. If this continued, players were eliminated from the test. The level and shuttle immediately prior to elimination from the test was recorded as the player's score. This score was used to estimate $\mathrm{VO}_{2 \max }{ }^{15}$ Players were instructed to cool down following the test with 10 minutes of easy jogging, walking and stretching.

\section{Repeated sprint testing}

A repeated sprint test was used to assess the ability to perform multiple bouts of high-intensity running between brief periods of rest. ${ }^{4}$ A level, even surface on a natural grass soccer pitch was used for the test with the subjects wearing full soccer kit. Six lines (marked 0 - 5) were placed at $5 \mathrm{~m}$ intervals. Players were required to sprint from the start line to line 1 , and back to the start line, then to line 2 , back to the start line, and so on. Players were instructed to sprint as far as they could in this fashion for $30 \mathrm{~s}$. A whistle blow signalled the start of each shuttle run, with six runs in total, each separated by $35 \mathrm{~s}$ of recovery time in which players returned to the start 
TABLE I. Anthropometrical, flexibility, power, strength-endurance, speed, agility, aerobic endurance, and repeat sprint data for players in successful $(N=70)$ and unsuccessful $(N=70)$ professional South African soccer teams

\begin{tabular}{|c|c|c|}
\hline Variable & Successful* (Mean \pm SD) & Unsuccessful $^{\dagger}$ (Mean \pm SD) \\
\hline Age (years) ${ }^{\ddagger}$ & $25.9 \pm 4.3$ & $23.7 \pm 3.9$ \\
\hline Mass (kg) & $73.7 \pm 9.1$ & $73.1 \pm 9.2$ \\
\hline Stature $(\mathrm{cm})$ & $176.3 \pm 7.1$ & $177.1 \pm 7.5$ \\
\hline Sum of seven skinfolds $(\mathrm{mm}) \S$ & $56.6 \pm 19.4$ & $56.0 \pm 17.9$ \\
\hline Body fat $(\%)^{\pi}$ & $13.3 \pm 3.5$ & $13.3 \pm 3.1$ \\
\hline Sit-and-reach (cm) & $40.2 \pm 8.4$ & $37.8 \pm 7.7$ \\
\hline Vertical jump (cm) & $54.6 \pm 8.5$ & $52.9 \pm 7.2$ \\
\hline Power (W/kg) II & $16.1 \pm 1.1$ & $15.9 \pm 1.1$ \\
\hline Maximum sit-ups in $2 \mathrm{~min}^{\star *}$ & $73 \pm 14$ & $78 \pm 13$ \\
\hline Maximum push-ups in $1 \mathrm{~min}$ & $44 \pm 10$ & $46 \pm 12$ \\
\hline Maximum pull-ups & $7 \pm 3$ & $7 \pm 4$ \\
\hline $10 \mathrm{~m}$ sprint time $(\mathrm{s})^{* *}$ & $1.86 \pm 0.07$ & $1.88 \pm 0.07$ \\
\hline $40 \mathrm{~m}$ sprint time $(\mathrm{s})^{* *}$ & $5.49 \pm 0.18$ & $5.57 \pm 0.23$ \\
\hline Illinois agility test time (s) & $16.29 \pm 0.45$ & $16.35 \pm 0.48$ \\
\hline Estimated $\mathrm{VO}_{2 \max }(\mathrm{ml} / \mathrm{kg} / \mathrm{min})^{\star \star} \mathrm{\dagger \dagger}^{\dagger}$ & $51.7 \pm 5.1$ & $53.5 \pm 4.8$ \\
\hline Total repeat sprint distance $(\mathrm{m})$ & $714 \pm 39$ & $716 \pm 37$ \\
\hline \multicolumn{3}{|c|}{${ }^{*}$ Finishing within the top four positions of the Professional Soccer League log. } \\
\hline \multicolumn{3}{|c|}{${ }^{\top}$ Finishing within the bottom six positions of the Professional Soccer League log. } \\
\hline \multicolumn{3}{|l|}{ FSignificantly different, $p<0.01$} \\
\hline \multicolumn{3}{|c|}{ §Skinfolds: triceps, subscapular, biceps, supra-iliac, calf, thigh, and abdominal. } \\
\hline \multicolumn{3}{|c|}{ "Based on the Siri formula as described by Lohman. ${ }^{16}$} \\
\hline \multicolumn{3}{|c|}{ "Based on vertical jump performance using the Lewis formula described by Fox and Mathews. ${ }^{10}$} \\
\hline \multicolumn{3}{|l|}{ "Significantly different, $p<0.05$} \\
\hline${ }^{\dagger \dagger}$ Based on performance in the $20 \mathrm{~m}$ Multi- & & \\
\hline
\end{tabular}

line. Strong verbal motivation was given and players were instructed to sprint as hard as possible. Total sprint distance for each $30 \mathrm{~s}$ sprint was recorded to the nearest $2.5 \mathrm{~m}$ and the distance of the six sprints was summed to obtain the total repeat sprint distance in metres.

\section{Data analysis}

Standard descriptive statistics (mean \pm standard deviation (SD)) were used to characterise the two sets of players. Unpaired independent $t$-tests were used to compare the results of the successful and unsuccessful group means. Results were considered significant at $p<0.05$.

\section{Results}

Table I presents the means \pm SD of the physical tests of players in the successful and unsuccessful groups.

No significant differences were found between the successful and unsuccessful groups in any of the anthropometrical and body composition measures, i.e. mass, stature, sum of seven skinfolds, and percentage body fat. There were also no statistically meaningful differences between players in the successful and unsuccessful groups in terms of sit-and-reach flexibility and vertical jump scores, even when the latter were expressed as power output per $\mathrm{kg}$ body mass $(16.1 \pm 1.1 \mathrm{~W} / \mathrm{kg}$ v. $15.9 \pm 1.1 \mathrm{~W} / \mathrm{kg})$. Similarly, agility performance $(16.29 \pm 0.45 \mathrm{~s}$ v. $16.35 \pm 0.48 \mathrm{~s})$ and total repeat sprint distance $(714 \pm 39 \mathrm{~m} \mathrm{v} .716 \pm 37 \mathrm{~m})$ were remarkably similar between the successful and less successful groups of players. Small and non-significant differences were found between groups for maximum pushups in 1 minute and maximum pull-ups.

Significant differences were found between groups on $10 \mathrm{~m}$ and $40 \mathrm{~m}$ sprint times in favour of the more successful teams. Differences were also found between groups in estimated $\mathrm{VO}_{2 \max }$ based on the $20 \mathrm{~m} \mathrm{MST}$, with the unsuccessful group of players achieving better aerobic endurance performances than players from the successful teams. It should be noted though that the reported standard error of estimation (SEE) for this test is $5.4 \mathrm{ml} / \mathrm{kg} / \mathrm{min}$, ${ }^{15}$ which is larger than the modest $1.8 \mathrm{ml} / \mathrm{kg} / \mathrm{min}$ difference between the means of these groups. The less-successful group also performed more sit-ups in 2 minutes than the successful players.

More significant $(p<0.01)$ differences were found between the groups in terms of age, with players in successful squads being on average $\sim 2$ years older than their less successful counterparts ( $25.9 \pm 4.3$ years v. $23.7 \pm 3.9$ years $)$. 


\section{Discussion}

Since players must move their own body mass during running, jumping and direction changes, a high body fat content would appear to be a disadvantage in soccer. ${ }^{17}$ Physical size could be theorised to be important in winning player contests during the game, but might negatively affect acceleration and nimbleness, arguably more valuable qualities in soccer. These players did appear lean and light, with both groups averaging $13.3 \%$ body fat, weighing $73.7 \pm 9.1 \mathrm{~kg}$ (successful) v. $73.1 \pm 9.2 \mathrm{~kg}$ (unsuccessful), and both having low sum of seven skinfolds $(56.6 \pm 19.4 \mathrm{~mm}$ v. $56.0 \pm 17.9 \mathrm{~mm}$ respectively). Significant differences in anthropometrical and body composition measures were not apparent between players in the different groups.

Players need explosive quickness and speed in making decisive runs in defence or attack. Explosive power, agility, and balance are often required in winning critical moments or contests within a game. Test results in this study were unable to discriminate between players in successful and lesssuccessful squads on the basis of lower back and hamstring flexibility (sit-and-reach test), strength endurance (maximum push-ups, sit-ups and pull-ups), and explosive power (vertical jump). These are widely performed physical fitness tests included in test batteries for sports performance, and soccer in particular. Although they may be valuable in assessing flexibility and musculoskeletal function for health-related fitness, it is possible that at the level of elite soccer players, the movement tasks are far removed from the requirements of an actual game.

Acceleration and running speed are among variables reportedly able to differentiate between levels and positions of play. ${ }^{24,26}$ During a soccer match, players generally sprint for average durations of less than 6 seconds. ${ }^{19}$ The $10 \mathrm{~m}$ and $40 \mathrm{~m}$ sprint tests appropriately target this duration of running work, and were therefore expected to highlight more successful players' ability to get from one point to another more quickly. Small significant differences (1.86 $\pm 0.07 \mathrm{v}$. $1.88 \pm 0.07 \mathrm{~s}$ and $5.49 \pm 0.18$ v. $5.57 \pm 0.23 \mathrm{~s}$ ) were found between groups on these components, but these arguably do not represent meaningful differences in performance.

Agility is the fitness component that describes the ability to change the direction of body motion rapidly, and results from a combination of a variety of physical components. ${ }^{24}$ Soccer players continuously change movement direction and body position during a match. The results of this study show no differences between players in successful and less-successful groups, and in fact, show remarkably similar values between these groups in agility performance. It is possible that this is a fundamental component required in soccer, no matter what the level of success.

The distance covered by outfield players approximates 10 - $13 \mathrm{~km}$ per game, ${ }^{23}$ with variations based on playing position, style of play, and match conditions. ${ }^{19}$ The vast majority (90 - 98\%) of the energy required during a soccer match is produced through oxidative metabolism. ${ }^{5,14}$ Edwards et al. ${ }^{8}$ suggest that elite-level soccer performance may be partly determined by aerobic capacity. It is reasonable to expect that elevations in aerobic power and capacity would help to sustain high work rates during a game ${ }^{19}$ and that teams with better aerobic endurance are more likely to exert their dominance, and ultimately be more successful. In the current study it is difficult to claim a meaningful difference between the groups considering that the statistically significant difference is much smaller than the error of measurement of the test, as mentioned above.

Players must be able to recover rapidly between intense bouts of work as the exercise pattern in soccer involves multiple sprints. ${ }^{19}$ This was assessed through the repeat sprint test. No significant differences were found between players from teams that were either successful or unsuccessful.

The results of this study seem to suggest that these common physical fitness tests were unable to discriminate between players of teams placed high or low on the PSL log. In other words, although the fitness scores of players in this study may be different from those of untrained individuals or players in different leagues, successful and unsuccessful teams within the same professional league could not be separated on the basis of physical fitness. Factors other than physical fitness may be more important in determining success in professional soccer, at least in South Africa. The most obvious of these include technical skill, tactical sense and ability, style of play, player motivation and frame of mind.

A number of limitations in the current study may warrant consideration. Body composition estimations may have been confounded by the wide ethnic influence in the squads. The outdoor testing was associated with ambient condition changes such as wind velocity, ambient temperature and relative humidity, which may well have affected sprint, repeat sprint, and endurance running performance. It is possible that the level of activity between players differed at the time of measurement in terms of content of early season training sessions, and that this influenced performance in the tests. Alternatively, other physical fitness tests that better replicate soccer requirements ${ }^{5}$ may produce more significant differences between successful and less-successful squads. Finally, these players were tested in the first half of the season, with changes in physical fitness status and squad profile likely during the course of a full season. Nonetheless, Reilly ${ }^{19}$ reported little fluctuation in physical fitness profiles of soccer players once the competitive season gets underway, as match play and general training maintain the training status at a relatively constant level.

Despite these potential limitations it is difficult to ignore that while no physical fitness parameter significantly separated higher and lower-ranked teams, the one measured parameter that may be associated with some form of technical experience - age - did show a highly significant difference between groups. Players in successful squads were on average 2.2 years older than in less-successful squads. It seems plausible that technical and tactical skill, and therefore 
team success would be better with the increased experience gained by older players.

\section{Conclusions}

These results support those of other studies of individual players in which the test results of non-elite players were comparable to those of elite players. ${ }^{22}$ It seems that in the South African professional soccer setting, league performance is not dependent on superior physical fitness within that league. As noted by Reilly et al., ${ }^{20}$ players may not require exceptional ability in any physical fitness component, but should possess a reasonably high ability in all areas. It may be that provided a particular fitness standard is achieved, factors such as technical skill, tactical sense and ability, style of play, and player motivation may contribute more to the difference in log position at the end of a season. It is possible that the factors most limiting further improvement in soccer performance are of a skill-type nature, and should be given preferential attention. This may be different from the situation in other countries, and is in contrast to the results of studies using players in overseas leagues. ${ }^{2,9,14,23,24,26}$ Managers, coaches, and trainers should consider this when planning training and conditioning sessions for teams. Emphasis on technical and tactical work within sessions and incorporating these elements into physical conditioning sessions may better target the preparation requirements in soccer. Further research should focus on whether other physical fitness tests or tests of technical skill more distinctly separate players in successful and less successful teams.

\section{Acknowledgements}

The author would like to thank the staff of the Institute for Sport Research, University of Pretoria, for their assistance in data collection; and the management, coaching staff, and players of the teams who participated in the study.

\section{REFERENCES}

1. Anonymous. Parreira: I want South African success. September 2006. www.fifa.com (last accessed 1 February 2007).

2. Åstrand PO, Rodahl K, Dahl HA, Strømme SB. Textbook of Work Physiology: Physiological Bases of Exercise. 4th ed. Champaign, III.: Human Kinetics, 2003.

3. Bangsbo J. The physiology of soccer: with special reference to intense intermittent exercise. Acta Physiol Scand 1994; 15: Suppl 619, 1-156.

4. Boddington MK, Lambert MI, St Clair-Gibson A, Noakes TD. Reliability of a 5-m shuttle test. J Sports Sci 2001; 19: 223-8.

5. Chamari $\mathrm{K}$, Hachana $\mathrm{Y}, \mathrm{Ahmed} \mathrm{YB}$, et al. Field and laboratory testing in young elite soccer players. Br J Sports Med 2004; 38:191-6.

6. Davis JA, Brewer J. Applied physiology of female soccer players. Sports Med 1993; 16: 180-9.

7. Durnin JVGA, Womersley J. Body fat assessed from total body density and its estimation from skinfold thickness: measurements on 481 men and women aged from 16 to 72 years. Br J Nutr 1974; 32: 77-97.

8. Edwards AM, MacFadyen AM, Clark N. Test performance indicators from a single soccer specific fitness test differentiate between highly trained and recreationally active soccer players. J Sports Med Phys Fitness 2003; 43: 14-20.

9. Ellis L, Gastin P, Lawrence S, et al. Protocols for the physiological assessment of team sport players. In: Gore CJ, ed. Physiological Tests for Elite Athletes. Lower Mitcham, Australia: Human Kinetics, 2000: 128-44.

10. Fox EL, Mathews DK. The Interval Training: Conditioning for Sports and General Fitness. Philadelphia: WB Saunders, 1974.

11. Getchell B. Physical Fitness: A Way of Life. 3rd ed. New York: MacMillan, 1985.

12. Hillis S. Preparations for the World Cup. Br J Sports Med 2000; 32: 95.

13. Hoeger WK. Principles and Labs for Physical Fitness and Wellness. Englewood, Colo.: Morton, 1991.

14. Hoff J. Training and testing physical capacities for elite soccer players. $J$ Sports Sci 2005; 23: 573-82.

15. Lèger LA, Lambert JA. Maximal multistage 20-m shuttle run test to predict $V_{2}$ max. Eur J Appl Physiol 1982; 49:1-12.

16. Lohman TG. Advances in Body Composition Assessment. Champaign, III.: Human Kinetics, 1992.

17. MacDougall, JD, Wenger HA. The purpose of physiological testing. In: MacDougall, JD, Wenger HA, Green HJ, eds. Physiological Testing of the High-performance Athlete. 2nd ed. Champaign, III.: Human Kinetics, 1991.

18. Norton K, Marfell-Jones M, Whittingham N, et al. Anthropometric assessment protocols. In: Gore CJ, ed. Physiological Tests for Elite Athletes. Lower Mitcham, Australia: Human Kinetics, 2000: 66-85.

19. Reilly T. Football. In: Reilly T, Secher N, Snell P, Williams C, eds. Physiology of Sports. London: E \& FN Spon, 1990: 371-425.

20. Reilly T, Bangsbo J, Franks A. Anthropometric and physiological predispositions for elite soccer. J Sports Sci 2000; 18: 669-83.

21. Roozen M. Action-reaction: Illinois agility test. NSCA's Performance Training Journal 2004; 3(5): 5-6.

22. Siegler J, Robergs $R$, Weingart $H$. The application of soccer performance testing protocols to the non-elite player. J Sports Med Phys Fitness 2006; 46: 44-51.

23. Stølen T, Chamari K, Castagna C, Wisløff U. Physiology of soccer: an update. Sports Med 2005; 35: 501-36.

24. Svensson M, Drust B. Testing soccer players. J Sports Sci 2005; 23: 60118.

25. Tossavainen M. Testing Athletic Performance in Team and Power Sports. Oulu, Finland: Newtest Oy, 2004.

26. Tumilty D. Protocols for the assessment of male and female soccer players. In: Gore CJ, ed. Physiological Tests for Elite Athletes. Lower Mitcham, Australia: Human Kinetics, 2000: 356-362.

27. Wilsey S. The beautiful game. National Geographic 2006; 209: 42-8. 